Pacific

Journal of

Mathematics

A COUNTEREXAMPLE TO THE CORONA THEOREM FOR OPERATORS ON $H^{2}\left(\mathbb{D}^{n}\right)$

E. Amar and C. Menini 


\title{
A COUNTEREXAMPLE TO THE CORONA THEOREM FOR OPERATORS ON $H^{2}\left(\mathbb{D}^{n}\right)$
}

\author{
E. Amar and C. Menini
}

\begin{abstract}
We built a counterexample to the corona theorem for operators in $H^{\infty}\left(\mathcal{L}\left(H^{2}\left(\mathbb{D}^{n-1}\right)\right)\right.$ for $n \geq 4$, which is close to a counterexample to the corona theorem for functions in the sense that the associated subspaces are invariant by all the shifts.
\end{abstract}

Let $\Omega$ be a domain in $\mathbb{C}^{n}$ and $H^{\infty}(\Omega)$ be the algebra of the bounded holomorphic functions in $\Omega$; we recall the corona problem:

Given $g_{1}, \ldots, g_{N} \in H^{\infty}(\Omega)$ such that:

$$
\forall z \in \Omega,\left|g_{1}(z)\right|^{2}+\cdots+\left|g_{N}(z)\right|^{2} \geq \delta^{2}>0,
$$

find $f_{1}, \ldots, f_{N} \in H^{\infty}(\Omega)$ such that:

$$
f_{1} g_{1}+\cdots+f_{N} g_{N}=1 .
$$

L. Carleson [3] proved this is possible in the case $n=1$ for the unit disc $\mathbb{D}$ in the complex plane $\mathbb{C}$ but the question is still open for $n>1$, even for as simple domains as the unit ball or the unit polydisc $\mathbb{D}^{n}$.

One can also consider the operators corona problem as follows [7], [13].

Let $E$ and $E^{\prime}$ be Hilbert spaces, $\mathcal{L}\left(E^{\prime}, E\right)$ the space of bounded linear operators from $E^{\prime}$ to $E$ and $H^{\infty}\left(\mathcal{L}\left(E^{\prime}, E\right)\right)$ the space of bounded holomorphic functions on the unit disc taking values in $\mathcal{L}\left(E^{\prime}, E\right)$, given $G \in$ $H^{\infty}\left(\mathcal{L}\left(E^{\prime}, E\right)\right)$ such that:

$$
\forall \lambda \in \mathbb{D}, \forall e \in E,\left\|G(\lambda)^{*} e\right\|^{2} \geq c^{2}\|e\|^{2}
$$

find $X \in H^{\infty}\left(\mathcal{L}\left(E, E^{\prime}\right)\right)$ such that:

$$
\forall \lambda \in \mathbb{D}, X(\lambda)^{*} G(\lambda)^{*}=1_{E} .
$$

The classical formulation of the operator corona problem given by Sz. Nagy is about left invertibility of analytic operator-valued functions and our formulation is about left invertibility of antianalytic operator-valued functions, the $G(\lambda)^{*}$.

However the function $\lambda \longrightarrow G(\bar{\lambda})^{*}$ being analytic the two formulations are equivalent, and the one we choose is best suited for our geometric point of view. 
For $\operatorname{dim} E<\infty, \operatorname{dim} E^{\prime}=1$, it is the Carleson corona problem for functions in the disc.

Using Carleson's result, Fuhrmann [4] proved the result for $\operatorname{dim} E^{\prime}<\infty$ and $\operatorname{dim} E<\infty$.

By a different method using a modification of the Wolff's proof of the corona theorem, Tolokonnikov [12] and Uchiyama ([7], appendice 3) independently, gave an affirmative answer in the case $\operatorname{dim} E \leq \infty, \operatorname{dim} E^{\prime}=1$.

Using Tolokonnikov-Uchiyama theorem $(\operatorname{dim} E=\infty)$, Vasyunin ([7], appendice 3) proved the result for $\operatorname{dim} E \leq \infty, \operatorname{dim} E^{\prime}<\infty$.

We emphasize that the results of Tolokonnikov-Uchiyama are scalar results while the ones by Fuhrmann-Vasyunin are matrix.

In the case when both spaces are infinitely dimensional, the answer is negative and a counterexample is given by Treil [13].

For us it is more convenient to split the function $G$ into $N$ parts $G=$ $\left(G_{1}, \ldots, G_{N}\right)$ and the formulation of the problem is now:

Given $G_{1}, \ldots, G_{N} \in H^{\infty}\left(\mathcal{L}\left(E^{\prime}, E\right)\right)$ such that:

$(\mathrm{OCH}) \quad \forall \lambda \in \mathbb{D}, \forall e \in E,\left\|G_{1}(\lambda)^{*} e\right\|^{2}+\cdots+\left\|G_{N}(\lambda)^{*} e\right\|^{2} \geq c^{2}\|e\|^{2}$

find $X_{1}, \ldots, X_{N} \in H^{\infty}\left(\mathcal{L}\left(E, E^{\prime}\right)\right)$ such that:

(OC)

$$
\forall \lambda \in \mathbb{D}, X_{1}(\lambda)^{*} G_{1}(\lambda)^{*}+\cdots+X_{N}(\lambda)^{*} G_{N}(\lambda)^{*}=1_{E} .
$$

In order to introduce our results, we need the following notations:

Let $H^{2}\left(\mathbb{D}^{n}\right)$ the Hardy space of the polydisc, i.e., with $\mathbb{T}:=\partial \mathbb{D}$ :

$$
\begin{aligned}
& H^{2}\left(\mathbb{D}^{n}\right):=\left\{f \text { holomorphic in } \mathbb{D}^{n}\right. \text { s.t. } \\
& \left.\qquad\|f\|_{2}^{2}:=\sup _{r<1} \int_{\mathbb{T}^{n}}|f(r \zeta)|^{2} d \sigma(\zeta)<\infty\right\} .
\end{aligned}
$$

If $E$ is a separable Hilbert space with orthonormal basis $\left\{\epsilon_{j}, j \in \mathbb{N}\right\}$ then we can define $[7]$ :

$$
\begin{array}{ll}
H^{2}(E):=\left\{f=\sum_{j \in \mathbb{N}} f_{j}(\cdot) \epsilon_{j} \text { s.t. } f_{j} \in H^{2}(\mathbb{D}) \quad\right. \text { and } & \\
\left.\qquad\|f\|_{2}^{2}:=\sum_{j \in \mathbb{N}}\left\|f_{j}\right\|_{2}^{2}<\infty\right\} .
\end{array}
$$

If $E=H^{2}\left(\mathbb{D}^{n-1}\right)$ then $H^{2}(E)$ can be seen as $H^{2}\left(\mathbb{D}^{n}\right)$.

For $E, E^{\prime}$ Hilbert spaces, $G(\cdot) \in H^{\infty}\left(\mathcal{L}\left(E^{\prime}, E\right)\right)$ then we know [7] that the subspace $G H^{2}\left(E^{\prime}\right)$ of $H^{2}(E)$ is invariant by the shift operator "multiplication by $z^{\prime \prime}: f \in H^{2}(E) \longrightarrow z f$.

One way to connect the corona problem for functions and the corona problem for operators is to associate to a function $g_{i}\left(z_{1}, z^{\prime}\right) \in H^{\infty}\left(\mathbb{D}^{n}\right), z^{\prime}=$ 
$\left(z_{2}, \ldots, z_{n}\right)$ the operator $G_{i}\left(z_{1}\right)$ acting on $E:=H^{2}\left(\mathbb{D}^{n-1}\right)$ by multiplication by $g_{i}\left(z_{1}, \cdot\right)$.

In this situation, all the shifts "multiplication by $z_{k}$ ", $k=1, \ldots, n$ operate on $H^{2}(E)$ and if $G_{i}$ is the operator of multiplication by a function $g_{i}\left(z_{1}, \cdot\right)$ then of course the subspace $G_{i} H^{2}(E)$ is invariant by multiplication by $z_{1}$ but also by all the other shifts.

We construct operator-valued functions $G_{1}, G_{2}$ on the unit disc (whose values are operators on $\left.H^{2}\left(\mathbb{D}^{n-1}\right), n \geq 4\right)$ satisfying Operator Corona Hypothesis $(\mathrm{OCH})$ but not satisfying the conclusion (OC). Theses operatorvalued functions $G_{i}, i=1,2$ are not just arbitrary ones, they are close in some sense to scalar functions in $H^{\infty}\left(\mathbb{D}^{n}\right)$ because the associated subspaces $G_{i} H^{2}\left(E_{i}\right) \subset H^{2}\left(\mathbb{D}^{n}\right)$ are invariant with respect to all the multiplication by $z_{k}, k=1, \ldots, n$.

Theorem 0.1. Let $E=H^{2}\left(\mathbb{D}^{n-1}\right), n \geq 4$, there exist $E_{i} \subset E i=1,2$ and $G_{i} \in H^{\infty}\left(\mathcal{L}\left(E_{i}, E\right)\right)$ such that $(\mathrm{OCH})$ is true but $(\mathrm{OC})$ is not.

Moreover the $G_{i} H^{2}\left(E_{i}\right) \subset H^{2}\left(\mathbb{D}^{n}\right), i=1,2$ are invariant by multiplication by all coordinates.

This result will be a special case in a general class of counterexamples (Theorem 2.1); its proof is given in Section 3. The key point to deduce Theorem 0.1 from Theorem 2.1 is the link between Bergman interpolating sequences, precisely caracterized by K. Seip [9] and unconditional systems of representing kernels in $H^{2}\left(\mathbb{D}^{n}\right)$ (these definitions are given in Sections 1 and 3$)$.

Our construction is inspired by the Treil's one but there are two important differences. The first one, and the most interesting for our point of view, is the invariance with respect to all shifts which is a necessary condition for operators coming from multiplication by scalar functions.

The second one is that Treil based his counterexample by making a sequence of vectors in a Hilbert space which is uniformly minimal but is not an unconditional system. The uniform minimality implies that $(\mathrm{OCH})$ is true and the non-unconditionality implies that (OC) is false. But in our case uniform minimality always implies unconditionality as we proved in Corollary 3.2 together with Theorem 4.1 and Corollary 4.2 .

Unfortunately in order to get a result for the corona problem for scalar functions in the polydisc the method must be refined. Operators that come from multiplication by scalar functions satisfying the corona hypothesis always satisfy the operator corona conclusion [2].

We want to thank the referee for his constructive critics and for pointing out interesting open questions (see Remark 4.4). 


\section{Interpolating sequences and unconditional system.}

If $\left\{e_{i}, i \in \mathbb{N}\right\}$ is a sequence of unit vectors in the Hilbert space $H$, we say that $\left\{e_{i}, i \in \mathbb{N}\right\}$ is uniformly minimal if there exists a constant $\delta>0$ such that:

$$
\forall i, \operatorname{dist}\left\{e_{i}, \operatorname{span}\left\{e_{k}, k \neq i\right\}\right\} \geq \delta,
$$

we say that $\left\{e_{i}, i \in \mathbb{N}\right\}$ is an unconditional system if there are two positive constants $A$ and $B$ such that:

$$
A^{2} \sum_{i}\left|\alpha_{i}\right|^{2} \leq\left\|\sum_{i} \alpha_{i} e_{i}\right\|^{2} \leq B^{2} \sum_{i}\left|\alpha_{i}\right|^{2} .
$$

This is equivalent to say that $\left\{e_{i}, i \in \mathbb{N}\right\}$ is an unconditional basis of $\operatorname{span}\left\{e_{i}, i \in \mathbb{N}\right\}$ and this is also equivalent to say that the collection of all moment sequences $\left\{\left\langle h, e_{i}\right\rangle, i \in \mathbb{N}\right\}$, for $h \in H$, coincide with the whole $l^{2}$ space.

To $a=\left(a_{1}, \ldots, a_{n}\right) \in \mathbb{D}^{n}$ we associate $k_{a}(z)$ the normalized reproducing kernel in $H^{2}\left(\mathbb{D}^{n}\right)$. If $n=1$ this is the normalized Cauchy kernel:

$$
a \in \mathbb{D}, z \in \mathbb{D}, k_{a}(z):=\frac{\sqrt{1-|a|^{2}}}{1-\bar{a} z},
$$

otherwise:

$$
k_{\left(a_{1}, \ldots, a_{n}\right)}\left(z_{1}, \ldots, z_{n}\right)=k_{a_{1}}\left(z_{1}\right) \ldots k_{a_{n}}\left(z_{n}\right) .
$$

We notice that the reproducing property gives, with $\mathbb{Z}$ the operator of multiplication by $z$ :

$\forall a \in \mathbb{D}, \forall h \in H^{2}(\mathbb{D}), a h(a)=\left\langle z h, k_{a}\right\rangle=\left\langle h, \mathbb{Z}^{*} k_{a}\right\rangle$ and $a h(a)=\left\langle h, \bar{a} k_{a}\right\rangle$, hence $\mathbb{Z}^{*} k_{a}=\bar{a} k_{a}$ which means that $k_{a}$ is an eigenvector for the backward shift.

The same if $e \in E, a \in \mathbb{D}$ then $k_{a} e$ is also an eigenvector for the backward shift in $H^{2}(E)$.

This implies immediately that the reproducing kernels in $H^{2}\left(\mathbb{D}^{n}\right)$ are eigenvectors for all backward shifts and this will be used later in Section 3.

Let $H=H^{2}\left(\mathbb{D}^{n}\right), \sigma:=\left\{a_{i}, i \in \mathbb{N}\right\} \subset \mathbb{D}^{n}$ and $\forall i \in \mathbb{N}, e_{i}=k_{a_{i}}$; saying that $\left\{e_{i}, i \in \mathbb{N}\right\}$ is an unconditional system in $H$ means that the operator $T_{\sigma}: H \longrightarrow \mathbb{C}^{\mathbb{N}}$ defined by:

$$
T_{\sigma} h:=\left\{\left\langle h, e_{i}\right\rangle, i \in \mathbb{N}\right\}=\left\{h\left(a_{i}\right) \sqrt{1-\left|a_{i}^{1}\right|^{2}} \cdots \sqrt{1-\left|a_{i}^{n}\right|^{2}}, i \in \mathbb{N}\right\}
$$

where $a_{i}=\left(a_{i}^{1}, \ldots, a_{i}^{n}\right) \in \mathbb{D}^{n}$, is continuous and onto $l^{2}(\mathbb{N})$.

This leads naturaly to the following definitions [1]:

Definition 1.1. We say that $\sigma:=\left\{a_{i}, i \in \mathbb{N}\right\} \subset \mathbb{D}^{n}$ is $H^{2}\left(\mathbb{D}^{n}\right)$ interpolating if the range of $T_{\sigma}$ contains $l^{2}(\mathbb{N})$; we say that $\sigma$ is strongly interpolating if moreover $T_{\sigma}$ is continuous from $H^{2}\left(\mathbb{D}^{n}\right)$ onto $l^{2}(\mathbb{N})$. 
If $n=1$, these two notions coincide [11]. If $n>1$ and $\sigma$ lies on the diagonal $\Delta$ of $\mathbb{D}^{n}, \Delta:=\left\{z=\left(z_{1}, \ldots, z_{n}\right) \in \mathbb{D}^{n}\right.$ s.t. $\left.z_{1}=\cdots=z_{n}\right\}$, then again these two notions coincide, as we shall prove it in Corollary 3.2, and we shall be mainly interested in that case.

\section{A general counterexample.}

Let $S_{1}:=\left\{e_{i}, i \in \mathbb{N}\right\}, S_{2}:=\left\{f_{i}, i \in \mathbb{N}\right\}$ be two sequences of unit vectors in $E$, an Hilbert space, and $\Gamma_{1}:=\left\{a_{i}, i \in \mathbb{N}\right\}, \Gamma_{2}:=\left\{b_{i}, i \in \mathbb{N}\right\}$ be two sequences of points in $\mathbb{D}$. Let $F_{1}:=\operatorname{span}\left\{k_{a_{i}} e_{i}, i \in \mathbb{N}\right\}, F_{2}:=\operatorname{span}\left\{k_{b_{i}} f_{i}, i \in\right.$ $\mathbb{N}\} \subset H^{2}(E)$, they are invariant subspaces with respect to the adjoint of the shift operator, because as we seen the $k_{a_{i}} e_{i}$ are eigenvectors for it; the same for $k_{b_{i}} f_{i}$.

Hence the orthogonal complement of $F_{i}$ in $H^{2}(E)$ are invariant by the shift and we can use the Beurling Halmos Lax theorem [7]:

For $i=1,2$, there exists subspaces $E_{i} \subset E$ and inner functions $G_{i} \in$ $H^{\infty}\left(\mathcal{L}\left(E_{i}, E\right)\right)$ such that the orthogonal complement of $F_{i}$ can be represented by $G_{i} H^{2}\left(E_{i}\right)$.

Here inner means that the boundary values of the operators $G_{i}$, which exist a.e. [7], are isometries, and the $G_{i}$ are unique up to a constant unitary function.

Of course we can assume $E_{i}=E$ provided we extend $G_{i}$ as 0 on $E \ominus E_{i}$, but then we loose the inner property.

This can be rewritten as $F_{i}=H^{2}(E) \ominus G_{i} H^{2}\left(E_{i}\right)$.

Recall that the Gleason (pseudo) distance in the unit disc $\mathbb{D}$ is

$$
\forall a, b \in \mathbb{D}, d_{g}(a, b):=\left|\frac{a-b}{1-\bar{a} b}\right| .
$$

Now we can state:

Theorem 2.1. If the following hypothesis are satisfied:

1. $S_{1}$ and $S_{2}$ are unconditional systems in $E$,

2. $\Gamma_{1}$ and $\Gamma_{2}$ are separated: $\exists \delta>0$ s.t. $\forall i \in \mathbb{N}, \forall j \in \mathbb{N}, d_{g}\left(a_{i}, b_{j}\right) \geq \delta$,

3. $\left\{k_{a_{i}} e_{i}, i \in \mathbb{N}\right\} \cup\left\{k_{b_{i}} f_{i}, i \in \mathbb{N}\right\}$ is not an unconditional system in $H^{2}(E)$, then we have for $G_{i}$ defined as above:

$$
\forall \lambda \in \mathbb{D}, \forall e \in E,\left\|G_{1}(\lambda)^{*} e\right\|^{2}+\left\|G_{2}(\lambda)^{*} e\right\|^{2} \geq c^{2}\|e\|^{2}
$$

but there are no operators $X_{i} \in H^{\infty}\left(\mathcal{L}\left(E, E_{i}\right)\right), i=1,2$ such that

$$
X_{1}(\lambda)^{*} G_{1}(\lambda)^{*}+X_{2}(\lambda)^{*} G_{2}(\lambda)^{*}=1_{E} .
$$

Points 1 and 2 will give us (OCH), Points 1 and 3 will give us that (OC) is false. 
2.1. Proof of Theorem 2.1: 1 and 2 imply (OCH). It will be an easy consequence of the following lemma:

Lemma 2.2. If $\left\|G_{1}(\lambda)^{*} e\right\|<t\|e\|$ for $0<t<1$ there exists $j_{0} \in \mathbb{N}$ such that $d_{g}^{2}\left(\lambda, a_{j_{0}}\right)<c(A, B) t$.

Proof of 1 and 2 imply $(\mathrm{OCH})$. Let $e \in E$ and suppose that $\left\|G_{i}(\lambda)^{*} e\right\|<$ $t\|e\|$ for $i=1,2$ and a $t$ such that $0<t<1$, then using the lemma above we get that there exist $j, k \in \mathbb{N}$ with

$$
d_{g}^{2}\left(\lambda, a_{j}\right)<c(A, B) t \text { and } d_{g}^{2}\left(\lambda, b_{k}\right)<c(A, B) t .
$$

Because $d_{g}$ verifies $d_{g}\left(a_{j}, b_{k}\right) \leq 2\left(d_{g}\left(a_{j}, \lambda\right)+d_{g}\left(\lambda, b_{k}\right)\right)$ we get a contradiction with the hypothesis of separation for $t$ small enough.

Proof of the lemma. We already know that ([13, Lemma 19.1, p. 269]):

$$
\left\|G_{1}(\lambda)^{*} e\right\|=\operatorname{dist}\left(k_{\lambda} e, F_{1}\right) .
$$

Let us assume $\|e\|=1$ and decompose $e \in E$ as:

$$
e=\sum_{i=1}^{\infty} \alpha_{i} e_{i}+e^{\perp} \text { with } e^{\perp} \text { orthogonal to span }\left\{e_{i}, i \in \mathbb{N}\right\},
$$

and let $P_{F_{1}}$ the orthogonal projection on $F_{1}, P_{F_{1}}\left(k_{\lambda} e\right)=\sum_{i=1}^{\infty} \beta_{i} k_{a_{i}} e_{i}$.

With (6) the hypothesis can be written

$$
\left\|\sum_{i=1}^{\infty}\left(\alpha_{i} k_{\lambda}-\beta_{i} k_{a_{i}}\right) e_{i}\right\|^{2}+\left\|k_{\lambda} e^{\perp}\right\|^{2}<t^{2} .
$$

Because the sequence $\left\{e_{i}, i \in \mathbb{N}\right\}$ is an unconditional system (7) implies:

$$
\sum_{i, \alpha_{i} \neq 0}\left|\alpha_{i}\right|^{2}\left\|k_{\lambda}-\frac{\beta_{i}}{\alpha_{i}} k_{a_{i}}\right\|^{2}<\frac{t^{2}}{A^{2}}
$$

Formula (7) implies also that $\left\|e^{\perp}\right\|^{2}<t^{2}$ but $\left\|\sum_{i=1}^{\infty} \alpha_{i} e_{i}\right\|^{2}=1-\left\|e^{\perp}\right\|^{2}$ and using again that $\left\{e_{i}, i \in \mathbb{N}\right\}$ is an unconditional system:

$$
\frac{1-t^{2}}{B^{2}}<\sum_{i}\left|\alpha_{i}\right|^{2} \leq \frac{1}{A^{2}}
$$

But now Formulas (8) and (9) imply that

$$
\exists j_{0} \in \mathbb{N}:\left\|k_{\lambda}-\frac{\beta_{j_{0}}}{\alpha_{j_{0}}} k_{a_{j_{0}}}\right\|^{2}<\frac{t^{2} B^{2}}{\left(1-t^{2}\right) A^{2}} .
$$


To simplify notations let us denote $\frac{\beta_{j_{0}}}{\alpha_{j_{0}}}$ by $\gamma, a_{j_{0}}$ by $\mu$ and $\frac{t^{2} B^{2}}{\left(1-t^{2}\right) A^{2}}$ by $s^{2}$. Then

$$
\begin{aligned}
\left\|k_{\lambda}-\gamma k_{\mu}\right\|_{H^{2}(\mathbb{D})}^{2} & =1+|\gamma|^{2}-2 \operatorname{Re}\left(\bar{\gamma}\left\langle k_{\lambda}, k_{\mu}\right\rangle\right), \\
\operatorname{Re}\left(\bar{\gamma}\left\langle k_{\lambda}, k_{\mu}\right\rangle\right) & =\operatorname{Re}\left(\gamma \epsilon \sqrt{1-d_{g}^{2}(\lambda, \mu)}\right), \quad \text { with }|\epsilon|=1,
\end{aligned}
$$

hence

$$
1+|\gamma|^{2}-2|\gamma| \sqrt{1-d_{g}^{2}(\lambda, \mu)}<s^{2} \Longrightarrow|1-| \gamma||<s ; \quad d_{g}^{2}(\lambda, \mu)<\frac{s^{2}}{1-s}
$$

and the conclusion.

Remark 2.3. We also proved that $e$ is "near" $e_{j_{0}}$.

2.2. Proof of Theorem 2.1: 1 and 3 imply (OC) false. Let us recall that if $F_{1}, F_{2}$ are two subspaces of $E$, the angle between $F_{1}$ and $F_{2}$ is the number $\alpha, 0 \leq \alpha \leq \frac{\pi}{2}$ such that:

$$
\cos \alpha=\sup _{x \in F_{1}, y \in F_{2}} \frac{|\langle x, y\rangle|}{\|x\|\|y\|} .
$$

Lemma 2.4. Let $\left\{h_{i}, i \in \mathbb{N}\right\},\left\{l_{i}, i \in \mathbb{N}\right\}$ be two unconditional systems in $E$ and $F_{1}:=\operatorname{span}\left\{h_{i}, i \in \mathbb{N}\right\}, F_{2}:=\operatorname{span}\left\{l_{i}, i \in \mathbb{N}\right\}$ then the angle between $F_{1}$ and $F_{2}$ is strictly positive if and only if $\left\{h_{i}, i \in \mathbb{N}\right\} \cup\left\{l_{i}, i \in \mathbb{N}\right\}$ is an unconditional system in $E$.

Proof. Let $x:=\sum_{i} \alpha_{i} h_{i}, y:=\sum_{i} \beta_{i} l_{i}$, then we get:

$$
\|x\|^{2}+\|y\|^{2}-2 \cos \alpha\|x\|\|y\| \leq\|x+y\|^{2} \leq\|x\|^{2}+\|y\|^{2}+2 \cos \alpha\|x\|\|y\|
$$

hence $\|x+y\|^{2} \leq 2\left(\|x\|^{2}+\|y\|^{2}\right)$. Now, without lost of generality, suppose that $\|x\| \geq\|y\|$ then:

$$
\begin{aligned}
\|x\|^{2}+\|y\|^{2}-2 \cos \alpha\|x\|\|y\| & =(\|y\|-\cos \alpha\|x\|)^{2}+\sin ^{2} \alpha\|x\|^{2} \\
& \geq \frac{\sin ^{2} \alpha}{2}\left(\|x\|^{2}+\|y\|^{2}\right)
\end{aligned}
$$

hence:

$$
\begin{aligned}
\frac{\sin ^{2} \alpha}{2} A^{2} \sum_{i}\left(\left|\alpha_{i}\right|^{2}+\left|\beta_{i}\right|^{2}\right) & \leq\left\|\sum_{i} \alpha_{i} h_{i}+\sum_{i} \beta_{i} l_{i}\right\|^{2} \\
& \leq 2 B^{2} \sum_{i}\left(\left|\alpha_{i}\right|^{2}+\left|\beta_{i}\right|^{2}\right)
\end{aligned}
$$

and the lemma. 
Proof of 1 and 3 imply (OC) false. $S_{1}$ unconditional system implies $\left\{k_{a_{i}} e_{i}\right.$, $i \in \mathbb{N}\}$ is an unconditional system because

$$
\left\|\sum_{j=1}^{\infty} \alpha_{j} k_{a_{j}} e_{j}\right\|^{2}=\int_{\partial \mathbb{D}}\left\|\sum_{j=1}^{\infty} \alpha_{j} k_{a_{j}}\left(e^{i \theta}\right) e_{j}\right\|_{E}^{2} \frac{d \theta}{2 \pi}
$$

hence

$$
\begin{aligned}
\int_{\partial \mathbb{D}} A^{2} \sum_{j}\left|\alpha_{j} k_{a_{j}}\left(e^{i \theta}\right)\right|^{2} \frac{d \theta}{2 \pi} & \leq\left\|\sum_{j} \alpha_{j} k_{a_{j}} e_{j}\right\|^{2} \leq \int_{\partial \mathbb{D}} B^{2} \sum_{j}\left|\alpha_{j} k_{a_{j}}\left(e^{i \theta}\right)\right|^{2} \frac{d \theta}{2 \pi} \\
A^{2} \sum_{j}\left|\alpha_{j}\right|^{2} & \leq\left\|\sum_{j} \alpha_{j} k_{a_{j}} e_{j}\right\|^{2} \leq B^{2} \sum_{j}\left|\alpha_{j}\right|^{2} .
\end{aligned}
$$

The two systems $\left\{k_{a_{i}} e_{i}, i \in \mathbb{N}\right\}$ and $\left\{k_{b_{i}} f_{i}, i \in \mathbb{N}\right\}$ are then unconditional.

The fact that $\left\{k_{a_{i}} e_{i}, i \in \mathbb{N}\right\} \cup\left\{k_{b_{i}} f_{i}, i \in \mathbb{N}\right\}$ is not an unconditional system implies by Lemma 2.4 that the angle between $F_{1}$ and $F_{2}$ is zero and by the following lemma of Treil, the norm of the operators $X_{i}$ cannot be bounded.

Lemma 2.5 (Treil [13], Lemma 17.1). Let $\Theta_{1}$ and $\Theta_{2}$ be inner functions in $H^{\infty}\left(\mathcal{L}\left(E_{1}, E\right)\right)$ and in $H^{\infty}\left(\mathcal{L}\left(E_{2}, E\right)\right)$ respectively. Then the angle between the spaces $K_{\Theta_{1}}:=H^{2}(E) \ominus \Theta_{1} H^{2}\left(E_{1}\right)$ and $K_{\Theta_{2}}:=H^{2}(E) \ominus \Theta_{2} H^{2}\left(E_{2}\right)$ is non-zero iff there exist two functions $\psi_{1} \in H^{\infty}\left(\mathcal{L}\left(E, E_{1}\right)\right)$ and $\psi_{2} \in$ $H^{\infty}\left(\mathcal{L}\left(E, E_{2}\right)\right)$ such that $\Theta_{1} \psi_{1}+\Theta_{2} \psi_{2}=I$.

Moreover the skew projection of $H^{2}(E)$ onto $K_{\Theta_{2}}$ with kernel $K_{\Theta_{1}}$ is equal to the least possible norm of such $\psi_{1}$ (or, indifferently $\psi_{2}$ ).

\section{The polydisc case.}

Let $\Gamma_{1}:=\left\{a_{i}, i \in \mathbb{N}\right\}$ and $\Gamma_{2}:=\left\{b_{i}, i \in \mathbb{N}\right\}$ be two sequences in the disc $\mathbb{D}$ and $S_{1}:=\left\{e_{i}:=k_{\left(a_{i}, a_{i}, a_{i}\right)}, i \in \mathbb{N}\right\}, S_{2}:=\left\{f_{i}:=k_{\left(b_{i}, b_{i}, b_{i}\right)}, i \in \mathbb{N}\right\}$ be the two sequences of reproducing kernels in $H^{2}\left(\mathbb{D}^{3}\right)$ of the points $\left(a_{i}, a_{i}, a_{i}\right)$ and $\left(b_{i}, b_{i}, b_{i}\right)$. In this section we shall prove that sequences $\Gamma_{i}, S_{i}, i=1,2$ exist verifying Points 1, 2 and 3 of Theorem 2.1 and this will imply a proof of Theorem 0.1.

Moreover the choice of $k_{a_{i}} e_{i}$ and of $k_{b_{i}} f_{i}$ automatically warranty the invariance of $G_{i} H^{2}\left(E_{i}\right)$ by multiplication by all the coordinates because these vectors are eigenvectors for all backward shifts.

We already seen the link between unconditional systems of reproducing kernels and $H^{2}\left(\mathbb{D}^{n}\right)$ strongly interpolating sequences and we shall state and 
use the link between $H^{2}\left(\mathbb{D}^{n}\right)$ interpolating sequences and interpolating sequences for weighted Bergman spaces $A_{\alpha}(\mathbb{D})$ defined by:

$$
\begin{aligned}
A_{\alpha}(\mathbb{D}):= \begin{cases}f & \text { holomorphic in } \mathbb{D}:\|f\|_{\alpha}^{2}\end{cases} \\
\left.:=\int_{\mathbb{D}}|f(z)|^{2}\left(1-|z|^{2}\right)^{2 \alpha-1} d m(z)<\infty\right\} .
\end{aligned}
$$

This link is provided by the following result:

Theorem 3.1 (C. Horowitz \& D. Oberlin [6]). For $n \geq 2$, the linear operator $D: H^{2}\left(\mathbb{D}^{n}\right) \longrightarrow A_{\frac{n-1}{2}}(\mathbb{D})$ defined by

$$
\forall f \in H^{2}\left(\mathbb{D}^{n}\right), \forall \zeta \in \mathbb{D}, D f(\zeta):=f(\zeta, \ldots, \zeta)
$$

is bounded and onto.

Corollary 3.2. The sequence $\left\{a_{i}^{\prime}:=\left(a_{i}, \ldots, a_{i}\right), i \in \mathbb{N}\right\} \subset \mathbb{D}^{n}$ is $H^{2}\left(\mathbb{D}^{n}\right)$ interpolating iff $\left\{a_{i}, i \in \mathbb{N}\right\} \subset \mathbb{D}$ is $A_{\frac{n-1}{2}}(\mathbb{D})$ interpolating.

Moreover in that case interpolating implies strongly interpolating.

Proof. First recall that $S^{\prime}:=\left\{a_{i}^{\prime}:=\left(a_{i}, \ldots, a_{i}\right), i \in \mathbb{N}\right\} \subset \mathbb{D}^{n}$ is $H^{2}\left(\mathbb{D}^{n}\right)$ interpolating means that:

$T_{S^{\prime}}: H^{2}\left(\mathbb{D}^{n}\right) \longrightarrow \mathbb{C}^{\mathbb{N}}$ defined by $T_{S^{\prime}} f:=\left\{\left(1-\left|a_{i}\right|^{2}\right)^{n / 2} f\left(a_{i}^{\prime}\right), i \in \mathbb{N}\right\}$ has $l^{2}(\mathbb{N})$ in its range, and $S:=\left\{a_{i}, i \in \mathbb{N}\right\} \subset \mathbb{D}$ is $A_{\frac{n-1}{2}}(\mathbb{D})$ interpolating means that:

$$
T_{S}: A_{\frac{n-1}{2}}(\mathbb{D}) \longrightarrow \mathbb{C}^{\mathbb{N}} \text { defined by } T_{S} f:=\left\{\left(1-\left|a_{i}\right|^{2}\right)^{n / 2} f\left(a_{i}\right), i \in \mathbb{N}\right\}
$$

has $l^{2}(\mathbb{N})$ in its range.

Now given $\left\{\lambda_{i}, i \in \mathbb{N}\right\}$ such that $\sum_{i \in \mathbb{N}}\left(1-\left|a_{i}\right|^{2}\right)^{n}\left|\lambda_{i}\right|^{2}<\infty$, if $S:=$ $\left\{a_{i}, i \in \mathbb{N}\right\} \subset \mathbb{D}$ is $A_{\frac{n-1}{2}}(\mathbb{D})$ interpolating there is a function $f \in A_{\frac{n-1}{2}}(\mathbb{D})$ such that $\forall i \in \mathbb{N}, f\left(a_{i}\right)=\lambda_{i}$, hence by the theorem of Horowitz \& Oberlin the surjectivity of the mapping gives that there is a function $F \in H^{2}\left(\mathbb{D}^{n}\right)$ such that $F(z, \ldots, z)=f(z)$ hence $\forall i \in \mathbb{N}, F\left(a_{i}, \ldots, a_{i}\right)=\lambda_{i}$, which means that $S^{\prime}$ is $H^{2}\left(\mathbb{D}^{n}\right)$ interpolating. The same for the other way, using this time the continuity of the mapping.

For weighted Bergman spaces an interpolating sequence is separated and this implies that the operator $T$ is continuous ([5, Lemma 5.19]), hence in this case interpolating sequence and strongly interpolating sequences coincide, thus we get the same for $H^{2}\left(\mathbb{D}^{n}\right)$ if the sequence lies in the diagonal of $\mathbb{D}^{n}$.

Now in order to have Conditions 1 to 3 of Theorem 2.1 we just have to found sequences $\Gamma_{1}=\left\{a_{i}, i \in \mathbb{N}\right\}$ and $\Gamma_{2}=\left\{b_{i}, i \in \mathbb{N}\right\}$ in $\mathbb{D}$, separated, such that for $i=1,2, \Gamma_{i}$ are interpolating sequences of $A_{\frac{3-1}{2}}(\mathbb{D})=A_{1}(\mathbb{D})$ 
in order to fill Condition 1 and such that $\Gamma_{1} \cup \Gamma_{2}$ is not interpolating for $A_{\frac{4-1}{2}}(\mathbb{D})=A_{3 / 2}(\mathbb{D})$ in order to have $3-$.

For this we shall use the following example by Seip [10] in the upper half plane $\mathbb{U}:=\{z \in \mathbb{C}: \operatorname{Im} z>0\}$ and we send it in the unit disc $\mathbb{D}$ by the Cayley transform. We note $A_{\alpha}(\mathbb{U})$ the image by the Cayley transform of $A_{\alpha}(\mathbb{D})$.

Theorem 3.3 (Seip [10], Th. 1.2). Let $\Gamma(a, b):=\left\{a^{m}(b n+i), n, m \in \mathbb{Z}\right\}$, then $\Gamma(a, b)$ is a set of interpolation for $A_{\alpha}(\mathbb{U})$ if and only if $b \ln a>\frac{2 \pi}{\alpha}$.

First of all one can remark that if $\left\{z_{n}, n \in \mathbb{N}\right\}$ is interpolating for $A_{\alpha}(\mathbb{U})$ then for any $c>0$ the sequence $\left\{c z_{n}, n \in \mathbb{N}\right\}$ is also interpolating for $A_{\alpha}(\mathbb{U})$ by the change of variables $z \longrightarrow \frac{z}{c}$.

Now choose $a$ and $b$ such that $b \ln a \leq \frac{4 \pi}{3}$ but $2 b \ln a>2 \pi$, then we have:

$\Lambda_{1}:=\left\{z_{n m}:=a^{2 m}(b n+i), n, m \in \mathbb{Z}\right\}$ is interpolating for $A_{1}(\mathbb{U})$ because $b \ln a^{2}>\frac{2 \pi}{1}$

$\Lambda_{2}:=\left\{z_{n m}:=a \times a^{2 m}(b n+i), n, m \in \mathbb{Z}\right\}$ is interpolating for $A_{1}(\mathbb{U})$ because $\Lambda_{2}=a \Lambda_{1}$,

but $\Lambda_{1} \cup \Lambda_{2}$ is not interpolating for $A_{\frac{3}{2}}(\mathbb{U})$ because $b \ln a \leq \frac{4 \pi}{3}$, which ends the proof of Theorem 0.1.

Remark 3.4. For $n=2$ we cannot have Theorem 2.1 when $S_{1}=\left\{k_{\alpha_{i}}, i \in\right.$ $\mathbb{N}\}$ and $S_{2}=\left\{k_{\beta_{i}}, i \in \mathbb{N}\right\}$ are unconditional systems of reproducing kernels in $H^{2}(\mathbb{D})$. Because in that case the sequences $\left\{\alpha_{i}\right\}$ and $\left\{\beta_{i}\right\}$ are interpolating sequences in $H^{2}(\mathbb{D})$ thus in $H^{\infty}(\mathbb{D})$. Then $\left\{\left(a_{i}, \alpha_{i}\right)\right\}$ and $\left\{\left(b_{i}, \beta_{i}\right)\right\}$ are interpolating sequences in $H^{\infty}\left(\mathbb{D}^{2}\right)$ and by Hypothesis 2 they are separated in $H^{\infty}\left(\mathbb{D}^{2}\right)$. The union of these two sequences is then interpolating for $H^{\infty}\left(\mathbb{D}^{2}\right)$, [14], hence strongly interpolating for $H^{2}\left(\mathbb{D}^{2}\right)([\mathbf{1}]$, Theorem 1$)$. Thus, Hypothesis 3 is violated and by Lemma 2.4, (OC) is always true.

Remark 3.5. By Corollary 3.2 if $S_{1}^{\prime}:=\left\{\left(a_{i}, a_{i}\right), i \in \mathbb{N}\right\} \subset \mathbb{D}^{2}$ is $H^{2}\left(\mathbb{D}^{2}\right)$ interpolating then $\Lambda_{1}:=\left\{a_{i}, i \in \mathbb{N}\right\} \subset \mathbb{D}$ is $A_{\frac{1}{2}}(\mathbb{D})$ interpolating. With the Seip's characterization, by mean of his density $D^{+}[\mathbf{9}]$, we have $D^{+}\left(\Lambda_{1}\right)<\frac{1}{2}$. The same if $S_{2}^{\prime}:=\left\{\left(b_{i}, b_{i}\right), i \in \mathbb{N}\right\}$ is $H^{2}\left(\mathbb{D}^{2}\right)$ interpolating then $\Lambda_{2}:=$ $\left\{b_{i}, i \in \mathbb{N}\right\} \subset \mathbb{D}$ is also $A_{\frac{1}{2}}(\mathbb{D})$ interpolating hence $D^{+}\left(\Lambda_{2}\right)<\frac{1}{2}$. Then $D^{+}\left(\Lambda_{1} \cup \Lambda_{2}\right)<\frac{1}{2}+\frac{1}{2}=1$ and again by Seip's characterization $\Lambda_{1} \cup \Lambda_{2}$ is $A_{1}(\mathbb{D})$ interpolating which is equivalent to $\left\{\left(a_{i}, a_{i}, a_{i}\right), i \in \mathbb{N}\right\} \cup\left\{\left(b_{i}, b_{i}, b_{i}\right)\right.$, $i \in \mathbb{N}\}$ is $H^{2}\left(\mathbb{D}^{3}\right)$ interpolating. This is why whe have to go up to $\mathbb{D}^{4}$ to get a counterexample by this method.

\section{Uniform minimality.}

Let us denote by $\phi_{a} \in A_{\alpha}(\mathbb{D})$ the normalized reproducing kernel of $a \in \mathbb{D}$ for the weighted Bergman space $A_{\alpha}(\mathbb{D})$. 
Theorem 4.1. The sequence $\left\{z_{j}\right\}_{j \in \mathbb{N}} \subset \mathbb{D}$ is interpolating for $A_{\alpha}(\mathbb{D})$ if and only if the associated sequence of normalized reproducing kernels $\left\{\phi_{z_{j}}\right\}_{j \in \mathbb{N}}$ is uniformly minimal in $A_{\alpha}(\mathbb{D})$.

Corollary 4.2. Let $S_{i}:=\left\{c_{i}, i \in \mathbb{N}\right\} \subset \mathbb{D}$ be a sequence of points in $\mathbb{D}$ such that the sequence $\left\{\phi_{c_{i}}, i \in \mathbb{N}\right\}$ is not uniformly minimal in $A_{\frac{n-1}{2}}(\mathbb{D})$ then the sequence $\left\{k_{\left(c_{i}, \ldots, c_{i}\right)}, i \in \mathbb{N}\right\}$ is not uniformly minimal in $H^{2}\left(\mathbb{D}^{n}\right)$.

Proof. It is a consequence of $k_{(c, \ldots, c)}=D^{*} \phi_{c}$ where $D$ is the bounded linear operator defined in Theorem 3.1.

Proof of Theorem 4.1. Let $\Phi_{a}(z)=\frac{a-z}{1-\bar{a} z}$ a conformal map exchanging $a$ and 0 and $G_{\Sigma}$ the unique solution of the extremal problem

$$
\sup \left\{\operatorname{Re} g(0): g\left(z_{j}\right)=0 \forall z_{j} \in \Sigma,\|g\|_{A_{\alpha}(\mathbb{D})} \leq 1\right\}
$$

where $\Sigma$ is a sequence not containing 0. The main result of Schuster-Seip [8] associated to the note following it, states:

Theorem 4.3 (Schuster \& Seip). The sequence $\Gamma=\left\{z_{j}\right\}_{j \in \mathbb{N}} \subset \mathbb{D}$ is interpolating for $A_{\alpha}(\mathbb{D})$ if and only if there is a $\delta>0$ such that $\forall k \in \mathbb{N}, G_{k}(0) \geq$ $\delta$, where:

$$
G_{k}(z):=G_{\Phi_{z_{k}}\left(\Gamma \backslash\left\{z_{k}\right\}\right)}(z) .
$$

If the sequence $\left\{z_{j}, j \in \mathbb{N}\right\}$ is interpolating for $A_{\alpha}(\mathbb{D})$ then $\left\{\phi_{z_{j}}, j \in \mathbb{N}\right\}$ is an unconditional system, hence it is uniformly minimal (U.M.).

For the converse we shall give the proof in our context $\alpha=\frac{n-1}{2}$.

Let $S:=\left\{e_{k}\right\}_{k \in \mathbb{N}}$ be unit vectors; saying that $S$ is U.M. is equivalent to say that there is a conjugate system $S^{*}:=\left\{h_{k}\right\}_{k \in \mathbb{N}}$ defined by $\left\langle h_{k}, e_{m}\right\rangle=\delta_{k, m}$ which verifies $\forall k,\left\|h_{k}\right\|_{\alpha} \leq M$.

The normalized reproducing kernels associated to the sequence $\Gamma=$ $\left\{z_{j}\right\}_{j \in \mathbb{N}} \subset \mathbb{D}$ are

$$
e_{j}=\sqrt{n-1} \frac{\left(1-\left|z_{j}\right|^{2}\right)^{n / 2}}{\left(1-\bar{z}_{j} z\right)^{n}} .
$$

First it is easy to see that if the sequence of vectors $\left\{e_{j}, j \in \mathbb{N}\right\}$ is U.M. then $\forall k \in \mathbb{N}, G_{k}(0) \neq 0$.

Moreover associated to the system $\left\{h_{k}\right\}_{k \in \mathbb{N}}$ one can construct functions $H_{k}$ (depending on a parameter $C$ ) defined by

$$
h_{k}(z)=\frac{\sqrt{n-1}}{C}\left\{\left(H_{k} \times\left(\Phi_{z_{k}}^{\prime}\right)^{1-n / 2}\right) \circ \Phi_{z_{k}}(z)\right\} \times \Phi_{z_{k}}^{\prime}(z)
$$

thus $H_{k}\left(\Phi_{z_{k}}\left(z_{j}\right)\right)=0 \forall j \neq k, H_{k}(0)=C$ and $\left\|h_{k}\right\|_{\alpha}^{2}=\frac{n-1}{C^{2}}\left\|H_{k}\right\|_{\alpha}^{2}$.

With $C=\frac{\sqrt{n-1}}{M}$ we have $\left\|H_{k}\right\|_{\alpha} \leq 1$ and by the maximal property of $G_{k}$, $\forall k \in \mathbb{N}, G_{k}(0) \geq \frac{\sqrt{n-1}}{M}>0$.

The sequence $\Gamma=\left\{z_{j}\right\}_{j \in \mathbb{N}} \subset \mathbb{D}$ is then interpolating by Theorem 4.3. 
Remark 4.4. An open interesting question pointed out by the referee is:

Does the uniform minimality of sequences of reproducing kernels $k_{\left(a_{i}, b_{i}\right)}$ in $H^{2}\left(\mathbb{D}^{2}\right)$ implies that it is an unconditional system even if $a_{i} \neq b_{i}$ ?

Moreover the study of the non-diagonal case may give an answer for the critical case of our method, $\mathrm{n}=3$ (i.e., Remark 3.5).

\section{References}

[1] E. Amar, Interpolation dans le polydisque de $\mathbb{C}^{n}$, Illinois J. Math., 24(3) (1980), 359-378, MR 82b:32017, Zbl 0449.32017.

[2] E. Amar and C. Menini, Interpolating sequences for Bergman spaces and the Corona problem for operators. Prepublication de Bordeaux, 1999.

[3] L. Carleson, Interpolation by bounded analytic functions and the corona problem, Ann. Math., 76 (1962), 547-559, MR 25 \#5186, Zbl 0112.29702.

[4] P. Fuhrmann, On the corona theorem and its applications to spectral problems in Hilbert spaces, Trans. Amer. Math. Soc., 132 (1968), 55-66, MR 36 \#5751, Zbl 0187.38002.

[5] H. Hedenmalm, B. Korenblum and K. Zhu, Theory of Bergman Spaces, Graduate Texts in Mathematics, 199, Springer, 2000, MR 2001c:46043, Zbl 0955.32003.

[6] C. Horowitz and D. Oberlin, Restriction of $H^{p}$ functions to the diagonal of $U^{n}$, Indiana University Math. J., 24 (1975), 767-772, MR 50 \#7583, Zbl 0294.32002.

[7] N.K. Nikolskii, Treatise on the Shift Operator, Grundlehren, 273, Springer-Verlag, 1986, MR 87i:47042, Zbl 0587.47036.

[8] A.P. Schuster and K. Seip, A Carleson type condition for interpolation in Bergman spaces, J. Reine Angew. Math., 497 (1998), 223-233, MR 99f:46034, Zbl 0916.30037.

[9] K. Seip, Beurling type density theorems in the unit disk, Invent. Math., 113 (1993), 21-39, MR 94g:30033, Zbl 0789.30025.

[10] _ Regular sets of sampling and interpolation for weighted Bergman spaces, Proc. Amer. Math. Soc., 117 (1993), 213-220, MR 93c:30051, Zbl 0763.30014.

[11] H.S. Shapiro and A.L. Shields, On some interpolation problems for analytic functions, Amer. J. Math., 83 (1961), 513-532, MR 24 \#A3280, Zbl 0112.29701.

[12] V.A. Tolokonnikov, Estimates in Carleson's corona theorem, Zap. Nauk. Sem. Leningrad (LOMI), 113 (1981), 178-198, MR 83d:46065, Zbl 0472.46024.

[13] S.R. Treil, Geometric methods in spectral theory of vector-valued functions: Some recent results, Oper. Theory Adv. Appl., 42 (1989), 209-280, MR 91j:47036, Zbl 0699.47009.

[14] N.Th. Varopoulos, Sur la réunion de deux compacts d'interpolation, C.R. Acad. Sci. Paris, 272 (1971), 950-952, MR 43 \#5312b, Zbl 0221.46048.

Received May 8, 2000 and revised February 1, 2002.

Université de Bordeaux I

M.P.B., 351 COURS DE LA LiBÉRATION

33405 TALEnCE

FRANCE 
E-mail address: Eric.Amar@math.u-bordeaux.fr

UNIVERSITÉ DE BordeAuX I

M.P.B., 351 COURS DE LA LibÉRATion

33405 Talence

FRANCE

E-mail address: Chantal.Menini@math.u-bordeaux.fr 